\title{
Surgical Early Complications of the Laparoscopic Insertion of Peritoneal Dialysis Catheter: A Cross-Sectional Descriptive Two-Center Study in Ahvaz, Southwest of Iran
}

\author{
Mohammad Ali Khaksar ${ }^{1 *}$, Fatemeh Hayati², Amin Bahreini ${ }^{3}$ Behrouz Shayesteh Zadeh³, Shokouh Shayan Pour²
}

\author{
'Students' Research Committee, Ahvaz Jundishapour University of Medical Sciences, Ahvaz, Iran. \\ ${ }^{2}$ Chronic Renal Failure Research Center, Jundishapour University of Medical Sciences, Ahvaz, Iran. \\ ${ }^{3}$ General Surgery Department, Jundishapour University of Medical Sciences, Ahvaz, Iran. \\ *Correspondence to: Mohammad Ali Khaksar (E-mail: khaksar.ma@ajums.ac.ir)
}

\begin{abstract}
Objectives: This study aimed to investigate the surgical complications of this technique of peritoneal dialysis catheter insertion in Imam Khomeini and Golestan hospitals in Ahvaz, southwest of Iran, in the period between 2007 and 2019.

Methods: In this retrospective cross-sectional analytic-descriptive study, we examined end-stage renal disease (ESRD) patients who underwent laparoscopic peritoneal dialysis catheter insertion between 2007 and 2019. Patients' records and follow-up information recorded in the hospital dialysis unit were reviewed and early infectious and mechanical complications of peritoneal dialysis catheter insertion were analyzed.

Results: In this study, 290 patients (144 males and 138 females) with ESRD who have a mean age of $46 \pm 19.7$ years (1-90 years) were recruited, of whom 8 patients were excluded due to incomplete information in their records, and 68 patients (24.1\%) had an early complication of surgery. Surgical complications included: Catheter exit-site infections in 20 patients (7.1\%), peritonitis in 18 patients (6.4\%), catheter exit-site leakage in 17 patients (6\%), catheter occlusion without migration in 16 patients (5.7\%), catheter migration in 7 patients (2.5\%), and hemoperitoneum in 7 patients (2.5\%).

Conclusion: Due to the low complications of laparoscopic surgery in the insertion of a peritoneal dialysis catheter, this technique is recommended as a safe surgical procedure with low complications.

Keywords: Peritoneal dialysis, early complications, infectious complications, mechanical complications
\end{abstract}

\section{Introduction}

There are several treatment methods for patients with kidney failure. These methods include hemodialysis, peritoneal dialysis, and kidney transplantation. Due to the limitations of hemodialysis and its complications such as the high prevalence of cardiovascular disease and hypertension, peritoneal dialysis has become more important. Peritoneal dialysis has been used to treat end-stage renal disease (ESRD) since 1976. This method has been used in Iran since 1995 as a kidney replacement therapy (KRT) along with other methods (hemodialysis and kidney transplantation), but the rate of its use has been much lower compared with hemodialysis. Various studies have shown that compared with hemodialysis, continuous ambulatory peritoneal dialysis (CAPD) provides a better quality of life for patients.

In peritoneal dialysis, an appropriate catheter is placed inside the peritoneal cavity. This catheter can be introduced to the patient's body by open surgery, through the skin, or by laparoscopy. Due to the complications of open surgery, today laparoscopic surgery for peritoneal dialysis catheter insertion has won great popularity owing to fewer complications compared with open surgery. However, the laparoscopic procedure has limited complications. ${ }^{1}$ In this study, we tried to investigate the complications of peritoneal dialysis catheter insertion surgery in Imam Khomeini and Golestan hospitals in Ahvaz, southwest of Iran in the period between 2007 and 2019.

\section{Methods}

This is a cross-sectional analytic-descriptive and retrospective study conducted in 2020 after obtaining approval from the
Ethics Committee of Ahvaz Jundishapur University of Medical Sciences (AJUMS). The study population included patients with end-stage renal disease who underwent laparoscopic peritoneal catheter (standard double cuffed straight Tenckhoff catheter) insertion performed by two experienced surgeons in Imam Khomeini and Golestan hospitals in Ahvaz between 2007 and 2019. As pre-operation care, patients were admitted to the general surgery ward and were then evaluated 24 hours before the scheduled time of surgery. Afterwards, under general anesthesia, pneumoperitoneum was achieved with a 10 -mm Hasson port through a vertical umbilical incision, and two 5-mm ports were placed for diagnostic laparoscopy and instrumentation. The peritoneal dialysis catheter was inserted so that the distal cuff was placed preperitoneally, with the catheter tip in the rectovesical pouch. Care was taken to ensure that the catheter followed a smooth curve to exit the abdomen in a lateral and downward direction. Also, for post-operation care, we observed patients for 24 hours, and in case there were no problems, they were discharged. Their dialysis was performed under the supervision of the peritoneal dialysis unit of Imam Khomeini Hospital in Ahvaz. The exclusion criterion of this study was incomplete patient record information.

All patient files between 2007 and 2019 were reviewed and written reports of the peritoneal dialysis unit of Imam Khomeini Hospital of Ahvaz were collected. Demographic information of patients included age and sex. In this study, the complications of laparoscopic insertion of peritoneal dialysis catheters were studied in Imam Khomeini and Golestan hospitals of Ahvaz. This study included early mechanical and 
infectious complications (the first 30 days) and included catheter migration and removal, catheter occlusion, catheter leakage, bleeding and peritonitis, and catheter exit-site infection.

\section{Statistical Analysis}

In the present study, data analysis was performed using SPSS ver. 23. Descriptive analysis of quantitative data involved central indices of mean and median as well as indices of variance dispersion, standard deviation and frequency tables.

\section{Results}

Laparoscopic catheterization had been performed on 290 patients from 2007 to 2019. Eight patients (5 females, 3 males) were excluded from the study due to incomplete information in their records. The patients included 144 males and 138 females, and the mean age of patients was $46 \pm 19.7$ years (age range between 1 and 90 years). Mortality and intraoperative complications were not seen in any of the patients. Out of the 282 patients, 214 patients (75.9\%), including 104 males and 110 females, did not have any complications, while the rest experienced at least one complication.

From among the patients investigated, 20 patients (7.1\%) had peritoneal dialysis catheter exit-site infection, including 8 males and 12 females. Peritonitis was observed in 18 patients (6.4\%), including 13 males and 5 females. The dialysis catheter of 4 patients was removed due to peritonitis. Among 18 patients with peritonitis, 10 patients had peritonitis with negative culture and were treated with antibiotics administered intraperitoneally. Four patients also developed peritonitis with Pseudomonas who were treated with intraperitoneal antibiotics. Three patients also developed peritonitis with Enterobacteriaceae culture, in one of whom peritoneal dialysis catheter was removed. Only one patient had peritonitis with hemolytic streptococcus culture, who was treated with intraperitoneal antibiotics, and finally, the catheter was removed.

Seventeen patients $(6 \%)$ had leakage of exit-site of the peritoneal catheter, of whom 10 were male and 7 were female. We observed that the catheter occlusion without catheter migration occurred in 16 patients (5.7\%), of whom 9 were male and 7 was female and underwent catheter removal. Seven patients $(2.5 \%)$ had peritoneal dialysis catheter migration, all of whom were male. Finally, 7 patients $(2.5 \%)$ had hemoperitoneum, of whom 4 were male and 3 were female.

Thus, in general, the most frequent early complication of peritoneal dialysis catheter insertion was a mechanical complication as opposed to infectious complications (16.7\% vs. $13.5 \%)$. The most common early mechanical complication was catheter leakage (6\%). Early infectious complications were exit-site catheter infection (7.1\%) and peritonitis (6.4\%) (Figure 1).

In the present study, $75.9 \%$ of the patients had no complications, while others had at least one complication (Table 1).

The results demonstrated that there was a significant association between catheter migration and gender (Table 2).

\section{Discussion}

The present study was a cross-sectional, retrospective, analytic-descriptive study that examined the prevalence of

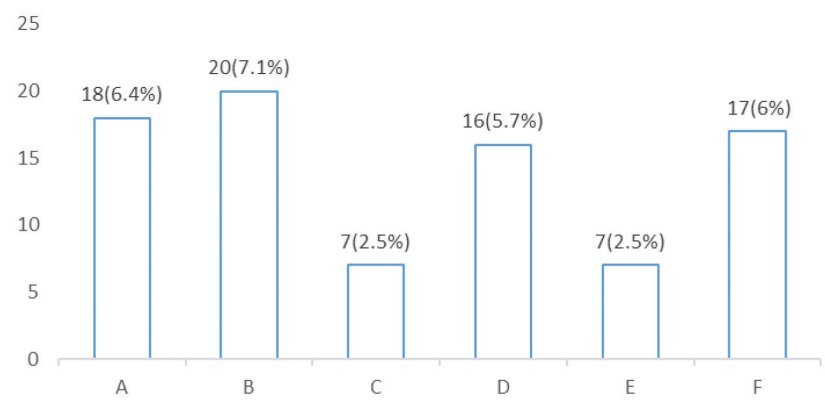

Fig. 1 Frequency of early complications of peritoneal dialysis catheter insertion. A: Peritonitis after catheter insertion B: Catheter exit-site infection C: Catheter migration D: Catheter occlusion without migration E: Intra-abdominal bleeding around the catheter F: Leakage of intra-abdominal fluid around the catheter.

\begin{tabular}{lcccc}
\hline \multicolumn{4}{l}{$\begin{array}{l}\text { Table 1. Frequency of early complications of peritoneal } \\
\text { dialysis catheter insertion }\end{array}$} \\
\hline Complication & Frequency & Percent & $\begin{array}{c}\text { Valid } \\
\text { percent }\end{array}$ & $\begin{array}{c}\text { Cumulative } \\
\text { percent }\end{array}$ \\
\hline 0 & 214 & 75.9 & 75.9 & 75.9 \\
1 & 55 & 19.5 & 19.5 & 95.4 \\
2 & 11 & 3.9 & 3.9 & 99.3 \\
3 & 2 & 0.7 & 0.7 & 100 \\
Total & 282 & 100 & 100 & \\
\hline
\end{tabular}

\begin{tabular}{lccl}
\hline \multicolumn{4}{l}{ Table 2. Complications and gender } \\
\hline Complications & Male $(\boldsymbol{n})$ & Female $(\boldsymbol{n})$ & \\
\hline Early peritonitis & 13 & 5 & 0.052 \\
Exit-site infection & 8 & 12 & 0.214 \\
Catheter migration & 7 & 0 & $0.008^{*}$ \\
Catheter occlusion & 9 & 7 & 0.434 \\
without migration & & & \\
Hemoperitoneum & 4 & 3 & 0.524 \\
Leakage of fluid & 10 & 7 & 0.342 \\
\hline
\end{tabular}

complications of laparoscopic peritoneal dialysis catheter surgery in ESRD patients in the first 30 days after surgery. In this study, overall, $24.1 \%$ of the patients experienced at least one early complication, which is in line with other studies which have reported the rate of such complications to be between $15 \%$ and $37.8 \% .^{2-4}$ By contrast, in García-Cruz et al., none of the patients who underwent laparoscopic peritoneal dialysis catheterization suffered from an early complication of peritoneal dialysis catheter insertion. ${ }^{5}$ The most frequent early complication of peritoneal dialysis catheter insertion in our study was mechanical complication (16.7\%). The most common early mechanical complication was catheter leakage (6\%). Early infectious complications were catheter exit-site infection (7.1\%) and peritonitis (6.4\%).

While most of the similar studies have compared the surgical complications of the two surgical methods of peritoneal dialysis catheter insertion surgery, ${ }^{6-10}$ there was no room for discussion regarding comparison of surgical methods since the only peritoneal dialysis catheter insertion method in our 
centers was the laparoscopic method. This method is more popular than open surgery method. One of the most important reasons for this is the lower damage in this technique of catheter insertion due to the proper view of the peritoneal cavity. As a result, there will be no complications such as perforation of the intestine or improper catheter placement, ${ }^{9,11,12}$ which were not observed in our study either.

One of the most important complications of catheter placement is infection, which is one of the main causes of catheter removal according to different studies. In the present study, the prevalence of catheter exit-site infection and that of peritonitis were $7.1 \%$ and $6.4 \%$, respectively, and the catheter of 4 patients was removed due to peritonitis. In this respect, Ashegh et al. reported that the most common early complications in their study were infectious complications and peritonitis, and that the catheter of 2 patients was removed due to peritonitis. Other studies have reported the rate of exit-site infection to be between $1.2 \%$ to $10 \% \cdot{ }^{1,2,13,14}$ Moreover, Keshvari et al. showed that the most common complication of peritoneal dialysis catheter insertion through open surgery was infectious complications, the most frequent of which was peritonitis. ${ }^{13}$ In comparison to open surgery, the incidence of infection is fewer in laparoscopic surgery, which might be due to the use of antibiotics before catheter placement, proper education of patients, and small surgical incision sites which can also reduce the risk of infection.

Displacement and migration of the catheter from the pelvis to other parts of the abdomen is another complication of catheter insertion which was seen in $2.5 \%$ of the patients, but it was found to be between 4 and $15 \%$ in other studies. ${ }^{1,4,14}$ This may be due to the use of a laparoscope to properly insert the catheter in place, in addition to other factors including the use of a low volume of dialysis fluid, the use of heparin after catheter placement, the low-volume changes in the first two weeks after catheterization, and the use of laxatives in patients to avoid constipation regardless of the type of surgical technique. ${ }^{15,16}$

Rate of fluid leakage from the catheter was $6 \%$ in our study, while it was reported to be between 1 and $17 \%$ in other studies. ${ }^{6,11,14,17}$ Of course, one study reported that none of their patients had fluid leakage. ${ }^{1}$ In Manouras et al., the prevalence of mechanical complications was $15.3 \%$, and the main complication was related to early leakage $(6 \%),{ }^{7}$ which is consistent with our study where the prevalence of mechanical complications was $16.7 \%$ and the major mechanical complication was catheter leakage with a rate of $6 \%$. The cause of less leakage in laparoscopic technique is the use of the appropriate laparoscopic technique with direct vision and less damage to the abdominal wall.

Another complication which was observed in our study was bleeding. This might be due to additional procedures performed in laparoscopic technique, such as the process of puncturing the abdominal wall by Trocar and suturing PD catheter to the abdominal cavity or peritoneum in laparoscopic surgery, fixation suture of the omentum, selective liver biopsy, and inguinal hernioplasties. ${ }^{18-20}$ Moreover, coagulation disorders may also cause bleeding.

As we stated earlier, the only peritoneal dialysis catheter insertion method in our centers was the laparoscopic technique, so we could not compare surgical complications in open versus laparoscopic methods. Since the period of our study involved 12 years, the records of some patients were incomplete, and information about comorbidities, BMI, etc. was not available.

Therefore more studies to evaluate the association between early complications of the laparoscopic insertion of peritoneal dialysis catheter and factors like comorbidities, BMI, and factors which can affect surgical complications are recommended. Also, we suggest that another study be conducted dealing exclusively with the late complications of the laparoscopic insertion of peritoneal dialysis catheter.

\section{Conclusion}

Considering that overall mechanical complications were more common than infectious complications in the present study, care must be exercised when using a peritoneal dialysis catheter. With a team consisting of experienced nephrologists, experienced surgeons, and well-trained nurses, along with appropriate patient education, the laparoscopic technique can be introduced as a reliable, safe, and uncomplicated method for inserting peritoneal dialysis catheters.

\section{Conflicts of Interest}

There are no conflicts of interest.

\section{References}

1. Musbahi A, Kanakala V. A modified laparoscopic peritoneal dialysis insertion technique, the 'one scar technique' can minimise short and long term complications: a retrospective cohort study. J Vasc Access. 2020:1129729820961970.

2. Ashegh H, Rezaii J, Esfandiari K, Roueentan A, Abouzari M. Laparoscopic placement of peritoneal dialysis catheters in CAPD patients: complications and survival. Tehran University Medical Journal TUMS Publications. 2008;66(3):186-90

3. Jwo SC, Chen KS, Lee CC, Chen HY. Prospective randomized study for comparison of open surgery with laparoscopic-assisted placement of Tenckhoff peritoneal dialysis catheter-a single center experience and literature review. J Surg Res. 2010;159(1):489-96.

4. Prabhakar N, Aljamal YN, Saleem HY, Baloul MS, Nyberg SL, Farley DR. Outcomes of laparoscopic and open CAPD catheter placement: a singlecenter experience. Surgery Open Science. 2019;1(1):20-4.

5. García-Cruz E, Vera-Rivera M, Molina JC, Mallafré-Sala J, Alcaraz A Laparoscopic placement of peritoneal dialysis catheter: description and results of a two-port. Official Publication of the Spanish Society of Nephrology. 2010;30(3):354-9.

6. Tsimoyiannis EC, Siakas P, Glantzounis G, Toli C, Sferopoulos G, Pappas M, et al. Laparoscopic placement of the Tenckhoff catheter for peritoneal dialysis. Surgical Laparoscopy Endoscopy \& Percutaneous Techniques. 2000;10(4):218-21.

7. Manouras AJ, Kekis PB, Stamou KM, Konstadoulakis MM, Apostolidis NS. Laparoscopic placement of Oreopoulos-Zellerman catheters in CAPD patients. Peritoneal Dialysis International. 2004;24(3):252-5

8. Chen Y, Shao Y, Xu J. The survival and complication rates of laparoscopic versus open catheter placement in peritoneal dialysis patients: a metaanalysis. Surg Laparosc Endosc Percutan Tech. 2015;25(5):440-3.

9. Li JR, Chen $\mathrm{CH}$, Cheng $\mathrm{CL}$, Yang $\mathrm{CK}$, Ou YC, Ho HC, et al. Five-year experience of peritoneal dialysis catheter placement. Journal of the Chinese Medical Association. 2012;75(7):309-13

10. van Laanen JHH, Cornelis T, Mees BM, Litjens EJ, van Loon MM, Tordoir JHM, et al. Randomized controlled trial comparing open versus laparoscopic placement of a peritoneal dialysis catheter and outcomes: the CAPD I trial. Perit Dial Int. 2018;38(2):104-12.

11. Bircan HY, Kulah E. Effects of a novel peritoneal dialysis: the open versus laparoscopic preperitoneal tunneling technique. Ther Apher Dial. 2016;20(1):66-72 
12. Shrestha BM, Shrestha D, Kumar A, Shrestha A, Boyes SA, Wilkie ME. Advanced laparoscopic peritoneal dialysis catheter insertion: systematic review and meta-analysis. Perit Dial Int. 2018;38(3): 163-71.

13. Keshvari A, Lesan Pezeshki M, Younesian M. Mechanical and infectious complications of peritoneal dialysis catheters in surgical wards of Imam Khomeini hospital: 7 year experience. Tehran University Medical Journal TUMS Publications. 2006;64(6):95-102.

14. Shahbandari M, Amiran A. Comparison of the complications of open surgery versus laparoscopic technique in insertion of peritoneal dialysis catheter. J Res Med Sci. 2019;24:85.

15. Shyr Y, Su C, Lui W. Complications of continuous ambulatory peritoneal dialysis: one surgeon's experience with 668 patient-month followup. Zhonghua yi xue za zhi = Chinese medical journal; Free China ed. 1995;55(4):307-14
16. Gadallah MF, Arora N, Arumugam R, Moles K. Role of Fogarty catheter manipulation in management of migrated, nonfunctional peritoneal dialysis catheters. American Journal of Kidney Diseases. 2000;35(2):301-5.

17. Özener Ç, Bihorac A, Akoglu E. Technical survival of CAPD catheters: comparison between percutaneous and conventional surgical placement techniques. Nephrology Dialysis Transplantation. 2001;16(9):1893-9.

18. Qiao Q, Lu G, Xu D, Zhou X, Li L. A comparison of two methods for catheterization in peritoneal dialysis. Jiangsu Med J. 2012;38(23):2812-4.

19. Öğünç G, Tuncer M, Öğünç D, Yardimsever M, Ersoy F. Laparoscopic omental fixation technique versus open surgical placement of peritoneal dialysis catheters. Surgical Endoscopy and Other Interventional Techniques. 2003;17(11):1749-55.

20. Xie P, Yuan L, Liu F. The comparison of efficacy and safety between laparoscopic and conventional placement of peritoneal dialysis catheters. Hebei Med. 2014;4:562-6.

This work is licensed under a Creative Commons Attribution-NonCommercial 3.0 Unported License which allows users to read, copy, distribute and make derivative works for non-commercial purposes from the material, as long as the author of the original work is cited properly. 